\title{
Constrained Detecting Arrays for Fault Localization in Combinatorial Testing
}

\author{
Hao Jin \\ Osaka University \\ Suita, Japan \\ k-kou@ist.osaka-u.ac.jp
}

\author{
Ce Shi \\ Shanghai Lixin University of \\ Accounting and Finance \\ Shanghai, China \\ shice060@lixin.edu.cn
}

\author{
Tatsuhiro Tsuchiya \\ Osaka University \\ Suita, Japan \\ t-tutiya@ist.osaka-u.ac.jp
}

\begin{abstract}
Detecting Arrays (DAs) are mathematical objects that enable fault localization in combinatorial interaction testing. Each row of a DA serves as a test case, whereas a whole DA is treated as a test suite. In real-world testing problems, it is often the case that some constraints exist among test parameters. In this paper, we show that it may be impossible to construct a DA using only constraintsatisfying test cases. The reason for this is that a set of some faulty interactions may always mask the effect of other faulty interactions in the presence of constraints. Based on this observation, we propose the notion of Constrained Detecting Arrays (CDAs) to adapt DAs to practical situations. The definition of CDAs requires that all rows of a CDA must satisfy the constraints and the same fault localization capability as the DA must hold except for such inherently undetectable faults. We then propose a computational method for constructing CDAs. Experimental results obtained by using a program that implements the method show that the method was able to produce CDAs within a reasonable time for practical problem instances.
\end{abstract}

\section{CCS CONCEPTS}

- Software and its engineering $\rightarrow$ Software testing and debugging; • Mathematics of computing $\rightarrow$ Combinatorial optimization.

\section{KEYWORDS}

Combinatorial interaction testing; fault localization; constraint handling; detecting arrays; SMT solver

\section{ACM Reference Format:}

Hao Jin, Ce Shi, and Tatsuhiro Tsuchiya. 2020. Constrained Detecting Arrays for Fault Localization in Combinatorial Testing. In The 35th ACM/SIGAPP Symposium on Applied Computing (SAC '20), March 30-April 3, 2020, Brno, Czech Republic. ACM, New York, NY, USA, Article 4, 8 pages. https://doi. org $/ 10.1145 / 3341105.3373952$

\section{INTRODUCTION}

Many testing strategies have been developed aiming at detecting and locating faults in systems and software. This paper focuses on

Permission to make digital or hard copies of part or all of this work for personal or classroom use is granted without fee provided that copies are not made or distributed for profit or commercial advantage and that copies bear this notice and the full citation on the first page. Copyrights for third-party components of this work must be honored For all other uses, contact the owner/author(s).

SAC '20, March 30-April 3, 2020, Brno, Czech Republic

(c) 2020 Copyright held by the owner/author(s).

ACM ISBN 978-1-4503-6866-7/20/03.

https://doi.org/10.1145/3341105.3373952 a particular testing strategy called Combinatorial Interaction Testing (CIT). CIT aims to detect faults caused by interactions among features or components and has been well practiced because of its effectiveness $[15,16]$.

Mathematical objects called Covering Arrays (CAs) play a key role in CIT. Roughly speaking, a CA of strength $t$ is a matrix where any $t$-way interaction appears in at least one row. A CA can be used as a test suite, where each row of the CA represents a test case. Since every $t$-way interaction occurs in some row of a CA, executing all test cases represented by the rows ensures executing all these interactions. When $t$ is small, CAs can be constructed by much fewer rows (test cases) than exhaustive test suites. Due to this property, using CAs as test suites significantly reduces testing cost while guaranteeing testing all $t$-way interactions.

Although CAs are sufficient for detecting the presence of a faulty interaction, the outcome obtained by executing CA-based test cases does not provide sufficient information for identifying the faulty interaction. To meet the need of locating faulty interactions, Colbourn and McClary introduced Detecting Arrays (DAs) in [3]. DAs are characterized by two integer parameters $d$ and $t$, where $d$ indicates the maximum number of faulty interactions that DAs can correctly locate and $t$ indicates the strength of interactions, that is, the number of elements composing each interaction.

The purpose of this paper is to propose an extended notion of DAs, which we call Constrained Detecting Arrays (CDAs), aiming at adapting DAs to real-world testing problems. Constraints among test parameters (representing components or features) are prevalent in practice [20,27,28]. Especially in highly configurable software systems, most of parameters usually have constraints. In fact, the notion of a CA is extended to the Constrained Covering Array (CCA) to reflect constraints on its definition. The CDA can be thought of a detecting array analog of CCA. However, extending the notion of DAs is not straightforward, because the fault localizing ability of DAs may no longer hold if there are constraints among test parameters. We circumvent this problem by relaxing the conditions on fault localization, resulting in the formal definition of CDAs. We also propose a method of constructing CDAs which uses a Satisfiability Modulo Theories (SMT) solver.

The remainder of the paper is organized as follows. Section 2 presents some basic notions including CAs and DAs. Section 3 describes constraints and introduces the definition of CDAs. Section 4 proposes an SMT-based CDA generation method. Section 5 shows the experimental results obtained using a program that implements the proposed generation method. Section 6 briefly surveys related work. Finally, Section 7 concludes this paper. 
Table 1: SUT Model of Mobile Phone System [2]

\begin{tabular}{l|lll}
\hline Parameter & \multicolumn{3}{|c}{ Values } \\
& $\left(v_{1}\right)$ & $\left(v_{2}\right)$ & $\left(v_{3}\right)$ \\
\hline$\left(F_{1}\right)$ Display & $16 \mathrm{MC}$ & $8 \mathrm{MC}$ & BW \\
$\left(F_{2}\right)$ Viewer & Graphical & Text & None \\
$\left(F_{3}\right)$ Camera & $2 \mathrm{MP}$ & $1 \mathrm{MP}$ & None \\
$\left(F_{4}\right)$ VideoCamera & Yes & No & \\
$\left(F_{5}\right)$ VideoRingtone & Yes & No \\
\hline
\end{tabular}

\section{PRELIMINARIES}

\subsection{SUT, Test Cases and Interactions}

We model the System Under Test (SUT) as a tuple $\mathcal{M}=\langle\mathcal{F}, \mathcal{S}\rangle$, where $\mathcal{F}=\left\{F_{1}, F_{2}, \ldots, F_{k}\right\}$ is the set of $k$ parameters and $\mathcal{S}=$ $\left\{S_{1}, S_{2}, \ldots, S_{k}\right\}$ is the set of domains for all the $k$ parameters, where $S_{i}$ is the domain for parameter $F_{i}$ and is the set of values from which $F_{i}$ can draw its value. Table 1 shows an example of a classical mobile phone system [2]. For presentation simplicity, we represent parameters as $F_{1}, F_{2}, \ldots$, instead of Display, Viewer, etc. in the rest of the paper. Similarly, we represent the values of the parameters as $v_{1}, v_{2}, \ldots$, instead of concrete value names.

A test case is a $k$-tuple that is an assignment of values to the $k$ parameters. That is, the $i$ th element in a test case $\sigma$ is a value $v_{j_{i}}$ chosen from the domain $S_{i}$ of the parameter $F_{i}$. For example, $\left(v_{3}, v_{1}, v_{3}, v_{1}, v_{2}\right)$ is a test case for the SUT model. Test cases can also be equivalently represented in the form as $\sigma=\left\{\left(F_{1}, v_{j_{1}}\right), \ldots\right.$, $\left.\left(F_{i}, v_{j_{i}}\right), \ldots,\left(F_{k}, v_{j_{k}}\right)\right\}(1 \leq i \leq k)$. The outcome of executing a test case is either pass or fail. A test case is passed or failed if its outcome is pass or fail, respectively.

A set of test cases is called a test suite. A test suite consisting of $N$ test cases can be regarded as an $N \times k$ array where each row corresponds to a test case. Throughout the paper, we use interchangeably the terms test suite and array.

An interaction is a set of parameter-value pairs such that all parameters are distinct. When the number of parameters involved in an interaction is $t$, we say that the interaction is $t$-way or that the strength of the interaction is $t$. For example, $\left\{\left(F_{1}, v_{3}\right),\left(F_{3}, v_{2}\right)\right\}$ is a two-way interaction or an interaction of strength two. This interaction corresponds to the pair of BW Display and 1MP Camera. In the special case when $t=0$, the interaction $T$ includes no parameters, i.e., $T=\emptyset$.

Clearly, an interaction $T$ is executed when a test case that has $T$ as its subset is executed. We say that an interaction $T$ is covered by a test case $\sigma$ and that $\sigma$ covers $T$ if and only if $T \subseteq \sigma$. When a test suite $A$ is given, the set of test cases that cover an interaction $T$ is denoted as $\rho_{A}(T)$, i.e., $\rho_{A}(T)=\{\sigma \mid T \subseteq \sigma \wedge \sigma \in A\}$. Since the interaction of strength $t=0$ is an empty set, it can be regarded as a subset of all test cases. In other words, all test cases cover the interaction of strength $t=0$. The definition of $\rho_{A}()$ is extended from an interaction $T$ to a set $\mathcal{T}$ of interactions as follows: We let $\rho_{A}(\mathcal{T})=\bigcup_{T \in \mathcal{T}} \rho_{A}(T)$.

We assume that an interaction is either faulty or not faulty and that the outcome of executing a test case $\sigma$ is fail if and only if $\sigma$ covers at least one faulty interaction.

\subsection{Covering Arrays (CAs)}

CAs are mathematical objects that can be used to exercise all interactions of a specific strength. When $t$ denotes the strength, CAs
Table 2: Two-Way CA for Mobile Phone System

\begin{tabular}{c|ccccc}
\hline$\sigma$ & $F_{1}$ & $F_{2}$ & $F_{3}$ & $F_{4}$ & $F_{5}$ \\
\hline 1 & $v_{1}$ & $v_{1}$ & $v_{2}$ & $v_{2}$ & $v_{2}$ \\
2 & $v_{1}$ & $v_{2}$ & $v_{3}$ & $v_{1}$ & $v_{1}$ \\
3 & $v_{1}$ & $v_{3}$ & $v_{1}$ & $v_{2}$ & $v_{1}$ \\
4 & $v_{2}$ & $v_{1}$ & $v_{3}$ & $v_{1}$ & $v_{2}$ \\
5 & $v_{2}$ & $v_{2}$ & $v_{1}$ & $v_{2}$ & $v_{2}$ \\
6 & $v_{2}$ & $v_{3}$ & $v_{2}$ & $v_{1}$ & $v_{1}$ \\
7 & $v_{3}$ & $v_{1}$ & $v_{1}$ & $v_{1}$ & $v_{1}$ \\
8 & $v_{3}$ & $v_{2}$ & $v_{2}$ & $v_{2}$ & $v_{2}$ \\
9 & $v_{3}$ & $v_{3}$ & $v_{3}$ & $v_{2}$ & $v_{2}$ \\
\hline
\end{tabular}

that are able to test all $t$-way interactions are called $t$-way CAs or $t$-CAs for short.

Definition 2.1. An $N \times k$ array $A$ is a $t$-CA if and only if the following condition is satisfied:

$$
\forall T \in \mathcal{I}_{t}: \rho_{A}(T) \neq \emptyset
$$

where $\mathcal{I}_{t}$ is a set of all $t$-way interactions.

Table 2 shows a 2-CA for the SUT model described in Table 1. All two-way interactions, 67 interactions in total, are covered by at least one row in the array. The CA is optimal with respect to the number of rows, because clearly there is no 2-CA with eight rows. By using the $\mathrm{CA}$ as a test suite and executing all the test cases, all two-way interactions are executed; thus the existence of a faulty interaction of strength two can be detected. For example, if an interaction between 16MC Display, $\left(F_{1}, v_{1}\right)$, and Graphical Viewer, $\left(F_{2}, v_{1}\right)$, is faulty, the outcome for test case $\sigma_{1}$ will be fail.

To test the SUT model with an exhaustive testing test suite, the tester would need to construct and execute $3 \times 3 \times 3 \times 2 \times 2=$ 108 test cases. In contrast, the 2-CA only contains 9 rows (test cases) as shown in Table 2. This example illustrates that using CAs can significantly reduce testing cost. In this particular case, the reduction rate is up to $92 \%$.

In addition to fault detection, fault localization is also practically important in testing. Failed test cases provide some clues for identifying a faulty interaction. For instance, consider the example shown in Table 2. If only the first test case $\sigma_{1}$ has failed, the interaction $\left\{\left(F_{1}, v_{1}\right),\left(F_{2}, v_{1}\right)\right\}$ can be suspected to be faulty. However, the interactions $\left\{\left(F_{1}, v_{1}\right),\left(F_{3}, v_{2}\right)\right\},\left\{\left(F_{1}, v_{1}\right),\left(F_{5}, v_{2}\right)\right\},\left\{\left(F_{2}, v_{1}\right),\left(F_{3}, v_{2}\right)\right\}$ and $\left\{\left(F_{2}, v_{1}\right),\left(F_{4}, v_{2}\right)\right\}$ are also covered only by the test case $\sigma_{1}$, which means that the test outcomes obtained from the CA do not provide enough information to judge which interaction is actually faulty.

\subsection{Detecting Arrays (DAs)}

In order to not only detect the existence of faulty interactions but also to locate them precisely, Colbourn and McClary proposed $(d, t)$-Detecting Arrays (DAs). If there exist $d$ or less $t$-way faulty interactions, a $(d, t)$-DA can determine their existence and locate which interactions are the faulty ones.

A $(d, t)$-DA is defined as follows.

Definition 2.2. An $N \times k$ array $A$ is a $(d, t)$-DA if and only if the following condition is satisfied:

$$
\forall \mathcal{T} \subseteq \mathcal{I}_{t} \text { s.t. }|\mathcal{T}|=d, \forall T \in \mathcal{I}_{t}: T \in \mathcal{T} \Leftrightarrow \rho_{A}(T) \subseteq \rho_{A}(\mathcal{T})
$$

where $\mathcal{I}_{t}$ is a set of all $t$-way interactions. 
Table 3: (1,2)-DA for Mobile Phone System

\begin{tabular}{c|ccccc}
\hline $\boldsymbol{\sigma}$ & $F_{1}$ & $F_{2}$ & $F_{3}$ & $F_{4}$ & $F_{5}$ \\
\hline 1 & $v_{1}$ & $v_{1}$ & $v_{1}$ & $v_{1}$ & $v_{1}$ \\
2 & $v_{1}$ & $v_{1}$ & $v_{2}$ & $v_{1}$ & $v_{2}$ \\
3 & $v_{1}$ & $v_{1}$ & $v_{3}$ & $v_{2}$ & $v_{2}$ \\
4 & $v_{1}$ & $v_{2}$ & $v_{1}$ & $v_{2}$ & $v_{2}$ \\
5 & $v_{1}$ & $v_{2}$ & $v_{3}$ & $v_{1}$ & $v_{1}$ \\
6 & $v_{1}$ & $v_{3}$ & $v_{1}$ & $v_{1}$ & $v_{2}$ \\
7 & $v_{1}$ & $v_{3}$ & $v_{2}$ & $v_{2}$ & $v_{1}$ \\
8 & $v_{2}$ & $v_{1}$ & $v_{1}$ & $v_{1}$ & $v_{2}$ \\
9 & $v_{2}$ & $v_{1}$ & $v_{2}$ & $v_{2}$ & $v_{1}$ \\
10 & $v_{2}$ & $v_{2}$ & $v_{2}$ & $v_{1}$ & $v_{2}$ \\
11 & $v_{2}$ & $v_{2}$ & $v_{3}$ & $v_{2}$ & $v_{1}$ \\
12 & $v_{2}$ & $v_{3}$ & $v_{1}$ & $v_{2}$ & $v_{1}$ \\
13 & $v_{2}$ & $v_{3}$ & $v_{2}$ & $v_{1}$ & $v_{1}$ \\
14 & $v_{2}$ & $v_{3}$ & $v_{2}$ & $v_{2}$ & $v_{2}$ \\
15 & $v_{2}$ & $v_{3}$ & $v_{3}$ & $v_{1}$ & $v_{2}$ \\
16 & $v_{3}$ & $v_{1}$ & $v_{1}$ & $v_{2}$ & $v_{2}$ \\
17 & $v_{3}$ & $v_{1}$ & $v_{3}$ & $v_{1}$ & $v_{1}$ \\
18 & $v_{3}$ & $v_{2}$ & $v_{1}$ & $v_{1}$ & $v_{1}$ \\
19 & $v_{3}$ & $v_{2}$ & $v_{2}$ & $v_{2}$ & $v_{1}$ \\
20 & $v_{3}$ & $v_{2}$ & $v_{3}$ & $v_{1}$ & $v_{2}$ \\
21 & $v_{3}$ & $v_{3}$ & $v_{2}$ & $v_{1}$ & $v_{2}$ \\
22 & $v_{3}$ & $v_{3}$ & $v_{3}$ & $v_{2}$ & $v_{1}$ \\
\hline & & & & &
\end{tabular}

This definition requires that if an interaction set $\mathcal{T}$ does not contain interaction $T^{\prime}$, then the set of test cases covering $T^{\prime}$, i.e., $\rho_{A}\left(T^{\prime}\right)$, must contain a test case that does not belong to $\rho_{A}(\mathcal{T})$, making $\rho_{A}(\mathcal{T})-\rho_{A}\left(T^{\prime}\right)$ nonempty. Consequently, every possible interaction set with size $d$, i.e., a combination of $d$ interactions, has a unique set of test cases in which at least one of the $d$ interactions is covered. As a result, the tester can determine the faulty interaction set from the failed test cases.

In the following, we illustrate how one can locate faulty interactions using a DA. Consider the (1,2)-DA in Table 3.

Now suppose that the test outcomes have been obtained from executing all the 22 test cases and that the outcomes of test cases $\sigma_{1}, \sigma_{2}$ and $\sigma_{3}$ are fail, whereas those of the other test cases are pass. By the definition of $(1,2)$-DAs, every two-way interaction has a unique set of test cases in which it is covered (note that we consider interaction sets of size one, as $d=1)$. In this case, $\left\{\left(F_{1}, v_{1}\right),\left(F_{2}, v_{1}\right)\right\}$ is the only interaction that is covered by the failed test cases and not covered by the passed test cases. As a result, this interaction can be determined as faulty.

If there are more than $d$ faulty $t$-way interactions, the set of failed test cases becomes distinct from any $\rho_{A}(\mathcal{T})$ such that $\mathcal{T} \subseteq \mathcal{I}_{t}$ and $\mathcal{T}=d$. For example, suppose that $\sigma_{1}, \sigma_{2}, \sigma_{3}$, and $\sigma_{4}$ are failed. There is no two-way interaction that is covered by all the four test cases. Hence, the tester can judge that there is more than one faulty interaction. On the other hand, if there are less than $d$ faulty interactions, these interactions can be determined by examining the failed and passed test cases. Further details can be found in [3].

\section{CONSTRAINED DETECTING ARRAYS}

In this section, constraints among parameters are described. Then, the notion of a Constrained Detecting Array (CDA), a variant of DA that incorporates constraints in its definition, is proposed.
Table 4: Mobile Phone System with Constraints

\begin{tabular}{l|lll}
\hline Parameter & \multicolumn{3}{|c}{ Values } \\
& $\left(v_{1}\right)$ & $\left(v_{2}\right)$ & $\left(v_{3}\right)$ \\
\hline$\left(F_{1}\right)$ Display & $16 \mathrm{MC}$ & $8 \mathrm{MC}$ & BW \\
$\left(F_{2}\right)$ Viewer & Graphical & Text & None \\
$\left(F_{3}\right)$ Camera & $2 \mathrm{MP}$ & $1 \mathrm{MP}$ & None \\
$\left(F_{4}\right)$ VideoCamera & Yes & No & \\
$\left(F_{5}\right)$ VideoRingtone & Yes & No & \\
\hline$\phi_{1}: F_{2}=v_{1} \Rightarrow F_{1} \neq v_{3}$ & \\
$\phi_{2}: F_{3}=v_{1} \Rightarrow F_{1} \neq v_{3}$ \\
$\phi_{3}: F_{2}=v_{1} \Rightarrow F_{3} \neq v_{1}$ \\
$\phi_{4}: F_{1}=v_{2} \Rightarrow F_{3} \neq v_{1}$ \\
$\phi_{5}: F_{4}=v_{1} \Rightarrow\left(F_{3} \neq v_{3} \wedge F_{1} \neq v_{3}\right)$ \\
$\phi_{6}: F_{5}=v_{1} \Rightarrow F_{4}=v_{1}$ \\
$\phi_{7}: \neg\left(F_{1}=v_{1} \wedge F_{2}=v_{2} \wedge F_{3}=v_{1}\right)$ \\
\hline
\end{tabular}

\subsection{Constraints}

In testing of real-world systems, some constraint among test parameters often arises. Such constraints can be originated from physical restrictions of the SUT, functional requirements, etc. Test cases that violate constraints are not executable, and thus must be avoided. A test case is said to be valid if it satisfies the constraints; invalid otherwise.

To describe constraints formally, we model each of them as a Boolean-valued formula $\phi_{i}$ consisting of parameters $F_{1}, F_{2}, \ldots, F_{k}$. The set of constraints is denoted as $\phi$, i.e., $\phi=\left\{\phi_{1}, \phi_{2}, \ldots\right\}$. An SUT with constraints can be represented with a 3-tuple $\mathcal{M}=\langle\mathcal{F}, \mathcal{S}, \phi\rangle$. Given an SUT $\mathcal{M}=\langle\mathcal{F}, \mathcal{S}, \phi\rangle$, the set of all valid test cases, denoted as $\mathcal{R}$, can be defined as follows.

$$
\begin{array}{r}
\mathcal{R}:=\left\{\boldsymbol{\sigma} \mid \boldsymbol{\sigma}=\left(v_{s_{1}}, v_{s_{2}}, \ldots, v_{s_{k}}\right) \in\left(S_{1} \times \cdots \times S_{k}\right) \wedge\right. \\
\left.\left.\bigwedge_{m=1}^{|\phi|} \phi_{m}\right|_{v_{s_{1}}, v_{s_{2}}, \ldots, v_{s_{k}}}=\text { true }\right\}
\end{array}
$$

where $\left.\phi_{m}\right|_{v_{s_{1}}, v_{s_{2}}, \ldots, v_{s_{k}}}$ denotes formula $\phi_{m}$ with each $F_{i}$ being replaced with $v_{s_{i}}$.

Table 4 shows the classical mobile phone system with constraints involved. The first constraint $\phi_{1}$, for example, represents the constraint that "The Graphical email viewer requires a color display" [2]. The second constraint $\phi_{2}$ means that "The 2 Megapixel camera requires a color display." In this example, test cases such as $\left(v_{1}, v_{1}, v_{2}, v_{2}, v_{1}\right.$ (representing (16MC, Graphical, 1MP, No, Yes)) and $\left(v_{2}, v_{1}, v_{1}, v_{1}, v_{1}\right)$ (representing (8MC, Graphical, 2MP, Yes, Yes)) are invalid, since they violate $\phi_{6}$ and $\phi_{4}$, respectively.

Invalid test cases result in interactions that cannot be executed. The notion of being valid or invalid can be naturally extended from test cases to interactions: If an interaction $T$ is covered by at least one valid test case $(\exists \sigma \in \mathcal{R}: T \subseteq \sigma)$, then the interaction is valid; otherwise, it is invalid. We let $\mathcal{V} \mathcal{I}_{t}$ denote the set of all valid $t$-way interactions. For example, $\left\{\left(F_{4}, v_{2}\right),\left(F_{5}, v_{1}\right)\right\}$ is an invalid interaction, since, because of $\phi_{6}$, there is no valid test case in which the interaction is covered.

We assume that faulty interactions are due to faults that are not related to constraint handling; thus we assume that faulty interactions, if any, are always valid interactions.

Covering arrays (CAs) can easily be adapted to incorporate constraints. This extended version of $\mathrm{CA}$ is the Constrained Covering Array (CCA). 
Definition 3.1. An $N \times k$ array $A$ is a $t$-CCA if and only if the following conditions are satisfied: All $N$ rows are valid test cases, i.e., $\forall i=1, \ldots, N: \sigma_{i} \in \mathcal{R}$; and

$$
\forall T \in \mathcal{V} \mathcal{I}_{t}: \rho_{A}(T) \neq \emptyset
$$

where $\sigma_{i}$ is the $i$ th row of $A$.

In contrast, incorporating constraints into Detecting Arrays (DAs) is not straightforward. As mentioned in Section 2.3, a $(d, t)$ DA $A$ requires that if a $t$-way interaction $T$ (a valid interaction, in this case) is not included in an interaction set $\mathcal{T}$, then $\rho_{A}(T) \nsubseteq$ $\rho_{A}(\mathcal{T})$ must hold. However, it may be impossible to implement this property when constraints exist. For instance, because of the constraint $\phi_{6}$ in Table 4, any valid test case that covers the interaction $T=\left\{\left(F_{1}, v_{1}\right),\left(F_{5}, v_{1}\right)\right\}$ must cover the interaction $T^{\prime}=$ $\left\{\left(F_{1}, v_{1}\right),\left(F_{4}, v_{1}\right)\right\}$. Hence, although $T \notin \mathcal{T}^{\prime}\left(\mathcal{T}^{\prime}=\left\{T^{\prime}\right\}\right), \rho_{A}(T) \subseteq$ $\rho_{A}\left(\mathcal{T}^{\prime}\right)$ always holds whenever the rows (test cases) of $A$ are all valid. This suggests that the fault localizing ability of the original DA needs to be compromised when constraints exist. Considering this aspect, we develop the definition of a constrained variant of DAs named Constrained Detecting Arrays (CDAs).

\subsection{Constrained Detecting Arrays (CDAs)}

In order to capture the necessary compromise in the fault localizing ability enforced by constraints, we define the notion of masking as follows:

Definition 3.2. An interaction set $\mathcal{T} \subseteq \mathcal{V} \mathcal{I}_{t}$ masks a valid interaction $T \in \mathcal{V} \mathcal{I}_{t}$ if and only if $T \notin \mathcal{T}$ and

$$
\forall \sigma \in \mathcal{R}: T \subseteq \sigma \Rightarrow\left(\exists T^{\prime} \in \mathcal{T}: T^{\prime} \subseteq \sigma\right)
$$

If $\mathcal{T}$ masks $T$, we write $\mathcal{T}>T$; otherwise we write $\mathcal{T} \nsucc T$.

Note that $\mathcal{R}$ is the set of all valid test cases and that $\mathcal{V} \mathcal{I}_{t}$ is the set of all valid interactions of strength $t(0 \leq t \leq k)$.

If $\mathcal{T}$ masks $T$ and all interactions in $\mathcal{T}$ are faulty, then whether $T$ is faulty or not cannot be decided using any set of valid test cases. By excluding such cases from the requirement on fault localization, we define Constrained Detecting Arrays (CDAs) as follows:

Definition 3.3. An $N \times k$ array $A$ is a $(d, t)$-CDA if and only if the following conditions are satisfied: The rows of $A$ are all valid test cases; and

$$
\begin{aligned}
\forall \mathcal{T} \subseteq \mathcal{V} \mathcal{I}_{t} \text { s.t. }|\mathcal{T}|=d, \forall T \in \mathcal{V} \mathcal{I}_{t}: \\
\qquad \mathcal{T} \ngtr T \Rightarrow\left(T \in \mathcal{T} \Leftrightarrow \rho_{A}(T) \subseteq \rho_{A}(\mathcal{T})\right)
\end{aligned}
$$

where $0 \leq d$ and $0 \leq t \leq k$.

Table 5 shows a $(1,2)$-CDA for the classical mobile phone system with constraints included. As mentioned in the previous section, the interaction set $\mathcal{T}^{\prime}=\left\{T^{\prime}\right\}=\left\{\left\{\left(F_{1}, v_{1}\right),\left(F_{4}, v_{1}\right)\right\}\right\}$ masks the interaction $T=\left\{\left(F_{1}, v_{1}\right),\left(F_{5}, v_{1}\right)\right\}$. It can be observed that $\rho_{A}(T)=$ $\left\{\sigma_{1}, \sigma_{3}, \sigma_{6}\right\}$ is a subset of $\rho_{A}\left(\mathcal{T}^{\prime}\right)=\left\{\sigma_{1}, \sigma_{3}, \sigma_{4}, \sigma_{6}, \sigma_{7}\right\}$. Hence, if $T^{\prime}$ is faulty, it is impossible to decide whether the $T$ is also faulty or not, and this is always the case whenever $A$ consists of valid test cases.

On the other hand, any interaction in $\mathcal{V} \mathcal{I}_{t}$ that is not masked by $\mathcal{T}^{\prime}$ can be safely removed from the candidates for faulty interactions. For example, $\left\{\left(F_{1}, v_{1}\right),\left(F_{2}, v_{1}\right)\right\}$ is covered by $\sigma_{2}$ which is not included in $\rho_{A}\left(\mathcal{T}^{\prime}\right)$.
Table 5: (1,2)-CDA for Mobile Phone System

\begin{tabular}{c|ccccc}
\hline $\boldsymbol{\sigma}$ & $F_{1}$ & $F_{2}$ & $F_{3}$ & $F_{4}$ & $F_{5}$ \\
\hline 1 & $v_{1}$ & $v_{1}$ & $v_{2}$ & $v_{1}$ & $v_{1}$ \\
2 & $v_{1}$ & $v_{1}$ & $v_{3}$ & $v_{2}$ & $v_{2}$ \\
3 & $v_{1}$ & $v_{2}$ & $v_{2}$ & $v_{1}$ & $v_{1}$ \\
4 & $v_{1}$ & $v_{2}$ & $v_{2}$ & $v_{1}$ & $v_{2}$ \\
5 & $v_{1}$ & $v_{2}$ & $v_{3}$ & $v_{2}$ & $v_{2}$ \\
6 & $v_{1}$ & $v_{3}$ & $v_{1}$ & $v_{1}$ & $v_{1}$ \\
7 & $v_{1}$ & $v_{3}$ & $v_{1}$ & $v_{1}$ & $v_{2}$ \\
8 & $v_{1}$ & $v_{3}$ & $v_{1}$ & $v_{2}$ & $v_{2}$ \\
9 & $v_{1}$ & $v_{3}$ & $v_{2}$ & $v_{2}$ & $v_{2}$ \\
10 & $v_{2}$ & $v_{1}$ & $v_{2}$ & $v_{1}$ & $v_{1}$ \\
11 & $v_{2}$ & $v_{1}$ & $v_{2}$ & $v_{1}$ & $v_{2}$ \\
12 & $v_{2}$ & $v_{1}$ & $v_{2}$ & $v_{2}$ & $v_{2}$ \\
13 & $v_{2}$ & $v_{1}$ & $v_{3}$ & $v_{2}$ & $v_{2}$ \\
14 & $v_{2}$ & $v_{2}$ & $v_{2}$ & $v_{1}$ & $v_{1}$ \\
15 & $v_{2}$ & $v_{2}$ & $v_{3}$ & $v_{2}$ & $v_{2}$ \\
16 & $v_{2}$ & $v_{3}$ & $v_{2}$ & $v_{1}$ & $v_{1}$ \\
17 & $v_{2}$ & $v_{3}$ & $v_{3}$ & $v_{2}$ & $v_{2}$ \\
18 & $v_{3}$ & $v_{2}$ & $v_{2}$ & $v_{2}$ & $v_{2}$ \\
19 & $v_{3}$ & $v_{2}$ & $v_{3}$ & $v_{2}$ & $v_{2}$ \\
20 & $v_{3}$ & $v_{3}$ & $v_{2}$ & $v_{2}$ & $v_{2}$ \\
21 & $v_{3}$ & $v_{3}$ & $v_{3}$ & $v_{2}$ & $v_{2}$ \\
\hline
\end{tabular}

Below we describe how to use the test outcomes of a $(d, t)$-CDA to spot a faulty interaction, using the $(1,2)$-CDA in Table 5 as an example. As $t=2$ for this example, we assume that faulty interactions, if any, are all two-way (of strength two). When there is at least one faulty interaction, the test outcomes can be divided into three cases.

The first case is where 1) the set of failed test cases matches or is contained in the set of test cases that covers some interaction set $\mathcal{T}$ of size $d$ and 2) $\mathcal{T}$ does not mask any interaction in $\mathcal{V} \mathcal{I}_{t}$. For example, if $\sigma_{1}$ and $\sigma_{2}$ are failed, $\mathcal{T}=\left\{\left\{\left(F_{1}, v_{1}\right),\left(F_{2}, v_{1}\right)\right\}\right\}$ is only such $\mathcal{T}$, as $\rho_{A}(\mathcal{T})=\left\{\sigma_{1}, \sigma_{2}\right\}$. As a result, it is able to judge that this is the only faulty interaction.

The second case is where 1) of the first case holds but 2) does not hold, that is, $\mathcal{T}$ masks some interaction in $\mathcal{V} \mathcal{I}_{t}$. For example, if $\boldsymbol{\sigma}_{6}, \boldsymbol{\sigma}_{7}, \boldsymbol{\sigma}_{8}$, and $\boldsymbol{\sigma}_{9}$ are failed, $\mathcal{T}=\left\{\left\{\left(F_{1}, v_{1}\right),\left(F_{2}, v_{3}\right)\right\}\right\}$ is the only interaction set that satisfies the first condition. Hence, $\left\{\left(F_{1}, v_{1}\right),\left(F_{2}, v_{3}\right)\right\}$ is determined to be faulty. However, $\mathcal{T}$ masks interaction $\left\{\left(F_{2}, v_{3}\right),\left(F_{3}, v_{1}\right)\right\}$; thus it is not possible to decide whether this interaction is also faulty or not.

The third case is where 1) of the above two cases does not hold; that is, the set of failed test cases does not match and not contained in $\rho_{A}(\mathcal{T})$ for any $\mathcal{T} \subseteq \mathcal{V} \mathcal{I}_{t}$ of size $d$. In this case, it is able to judge that there are more than $d$ faulty interactions. For example, if $\sigma_{18}, \sigma_{19}, \sigma_{20}$, and $\sigma_{21}$ are failed, it can be concluded that there is more than $d=1$ faulty interaction. Although it is impossible to identify the exact set of faulty interactions in this case, it is possible to narrow down the candidates for faulty interactions to those that appear only in these failed test cases.

\section{THE SMT-BASED GENERATION METHOD}

\subsection{Reduction to Satisfiability Problem}

In this section, a computation method is proposed to generate CDAs. In the proposed method, the problem of generating a CDA of a given 
number of rows (test cases) is reduced to the problem of checking the satisfiability of a logical (i.e., Boolean-valued) expression. A logical expression is satisfiable if it evaluates to true for some assignment of values to the variables. The proposed method creates a logical expression that is satisfiable if and only if a CDA of a given size exists. The expression is formulated so that the satisfying assignment of values to variables naturally represents the existing CDA. To check the satisfiability of the expression, we use an SMT (Satisfiability Modulo Theories) solver.

There are some existing studies on how to represent combinatorial designs, a class of discrete mathematical objects including CAs and DAs, using variables and logical expressions. In this paper, the naïve matrix model which is developed by Hnich et al. [10] is employed. In this model, an $N \times k$ array is represented as an $N \times k$ matrix of integer variables as follows.

$$
A=\left(\begin{array}{ccc}
p_{1}^{1} & \cdots & p_{k}^{1} \\
\vdots & \ddots & \vdots \\
p_{1}^{N} & \cdots & p_{k}^{N}
\end{array}\right)
$$

In the matrix, each variable $p_{i}^{n}$ represents the value of the parameter $F_{i}$ in the $n$-th test case $\sigma_{n}$. The domain of $p_{i}^{n}$ is $\left\{0,1, \ldots,\left|S_{i}\right|-1\right\}$. (Note that $\left|S_{i}\right|$ is the number of values that parameter $F_{i}$ can take.)

In order for the array $A$ to become a $(d, t)$-CDA, we impose the following conditions on $A$ using logical expressions.

(1) All rows (test cases) of $A$ are valid;

(2) Every pair of a $t$-way valid interaction $T$ and a set $\mathcal{T}$ of $d$ $t$-way valid interactions satisfies expression (6); and

(3) All $t$-way valid interactions are covered by at least one test case.

Clearly, the first and second conditions directly correspond to the two conditions that an array is a $(d, t)$-CDA. The third condition is used for practical purposes, since this ensures the resulting $(d, t)$ CDA is also a CCA.

In the rest of the section, we first present logical expressions that represent the above conditions. By logically ANDing all the expressions, we obtain a single logical expression which is in turn checked against satisfiability. Then we present the whole algorithm to generate $(d, t)$-CDAs. This algorithm repeatedly solves the satisfiability problem by decreasing the given array size one by one.

Valid Test Cases Consider the $n$-th row (test case). In $A$, this is expressed as a tuple of $k$ variables $\left(p_{1}^{n}, p_{2}^{n}, \ldots, p_{k}^{n}\right)$. As defined in Section 3, a test case is valid if and only if it satisfies all of the constraints and each of the constraint is the Boolean-valued

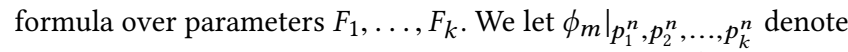
a constraint $\phi_{m}$ with each $F_{i}$ being replaced with $p_{i}^{n}$. Then, the following expression enforces the $n$-th test case to be valid.

$$
\operatorname{ValidTest}(n):=\left.\bigwedge_{m=1}^{|\phi|} \phi_{m}\right|_{p_{1}^{n}, p_{2}^{n}, \ldots, p_{k}^{n}}
$$

As a result, the following expression enforces $A$ to only contain valid test cases.

$$
\text { ValidSuite }:=\bigwedge_{n=1}^{N} \operatorname{ValidTest}(n)
$$

Representing Expression (6) The second condition holds if and only if Expression (6) is true for every $\mathcal{T}-T$ pair. That is,

$$
T \in \mathcal{T} \Leftrightarrow \rho_{A}(T) \subseteq \rho_{A}(\mathcal{T})
$$

or equivalently

$$
T \notin \mathcal{T} \Leftrightarrow \rho_{A}(T) \nsubseteq \rho_{A}(\mathcal{T})
$$

holds unless $\mathcal{T}>T$. This condition can be simplified as

$$
T \notin \mathcal{T} \Rightarrow \rho_{A}(T) \nsubseteq \rho_{A}(\mathcal{T})
$$

because the other direction trivially holds.

If $T \in \mathcal{T}$, this condition trivially holds. Hence we can focus on the case where $T \notin \mathcal{T}$ and $\mathcal{T} \nsucc T$. The right part of this formula, that is, $\rho_{A}(T) \nsubseteq \rho_{A}(\mathcal{T})$ is represented by a logical expression as follows:

$$
\begin{aligned}
& \operatorname{Locating}(\mathcal{T}, T):= \\
& \bigvee_{n=1}^{N}\left(\bigwedge_{j=1}^{t}\left(p_{x_{j}}^{n}=v_{x_{j}}\right) \wedge \neg\left(\bigvee_{L=1}^{d} \bigwedge_{l=1}^{t}\left(p_{y_{L_{l}}}^{n}=v_{y_{L_{l}}}\right)\right)\right)
\end{aligned}
$$

where $\mathcal{T}=\left\{\left\{\left(F_{y_{1_{1}}}, v_{y_{1_{1}}}\right), \ldots,\left(F_{y_{1_{t}}}, v_{y_{1_{t}}}\right)\right\}, \ldots,\left\{\left(F_{y_{d_{1}}}, v_{y_{d_{1}}}\right), \ldots\right.\right.$, $\left.\left.\left(F_{y_{d_{t}}}, v_{y_{d_{t}}}\right)\right\}\right\}$ and $T=\left\{\left(F_{x_{1}}, v_{x_{1}}\right), \ldots,\left(F_{x_{t}}, v_{x_{t}}\right)\right\}$. This expression ensures that there is at least one test case in $A$ that covers the interaction $T$ but does not cover any interaction in $\mathcal{T}$. Hence, if this is true, $\rho_{A}(T)$ will never be a subset of $\rho_{A}(\mathcal{T})$.

Covering Valid Interactions Given a valid interaction $T$, the following expression describes that the array $A$ includes at least one test case that covers $T$.

$$
\operatorname{Covering}(T):=\bigvee_{n=1}^{N} \bigwedge_{j=1}^{t}\left(p_{i_{j}}^{n}=v_{i_{j}}\right)
$$

The OR operator $\bigvee$ ensures $T$ to appear in at least one test case.

The Whole Expression The whole expression that will be checked against its satisfiability is obtained by conjuncting the expressions defined above as follows:

$$
\begin{aligned}
& \text { ValidSuite } \wedge \\
& \bigwedge_{\substack{\mathcal{T}, T: T \in \mathcal{V} \mathcal{I}_{t}, \mathcal{T} \subseteq \mathcal{T} \mathcal{T} \\
\mathcal{T} \ngtr T, T \notin \mathcal{T}},|\mathcal{T}|=d,} \operatorname{Locating}(\mathcal{T}, T) \wedge \\
& \bigwedge_{T \in \mathcal{V} I_{t}} \operatorname{Covering}(T)
\end{aligned}
$$

By checking the satisfiability of this expression, it can be checked whether or not a $(d, t)$-CDA of size $N$ exists or not. If it is satisfiable, then such a CDA exists. In this case, the satisfying assignment of integer values to $N \times k$ variables $p_{i}^{n}$ represents all the entries of one such CDA. On the other hand, if the expression is unsatisfiable, then it can be concluded that no $(d, t)$-CDA of size $N$ exists.

There are still problems that must be addressed in order to construct Expression (12). Specifically, we need to obtain $\mathcal{V} \mathcal{I}_{t}$ (see the subscripts of the two $\bigwedge$ ) and enumerate pairs of interaction set $\mathcal{T} \subseteq \mathcal{V} \mathcal{I}_{t}$ and interaction $T \in \mathcal{V} \mathcal{I}_{t}$ such that $|\mathcal{T}|=d, \mathcal{T} \ngtr T$, and $T \notin \mathcal{T}$ (see the subscripts of the first $\Lambda$ ). These problems can also be solved using satisfiability solving. We let integer variables $p_{1}, p_{2}, \ldots, p_{k}$ to symbolically represent a test case $\sigma$; that is,

$$
\sigma=\left(p_{1}, p_{2}, \ldots, p_{k}\right)
$$




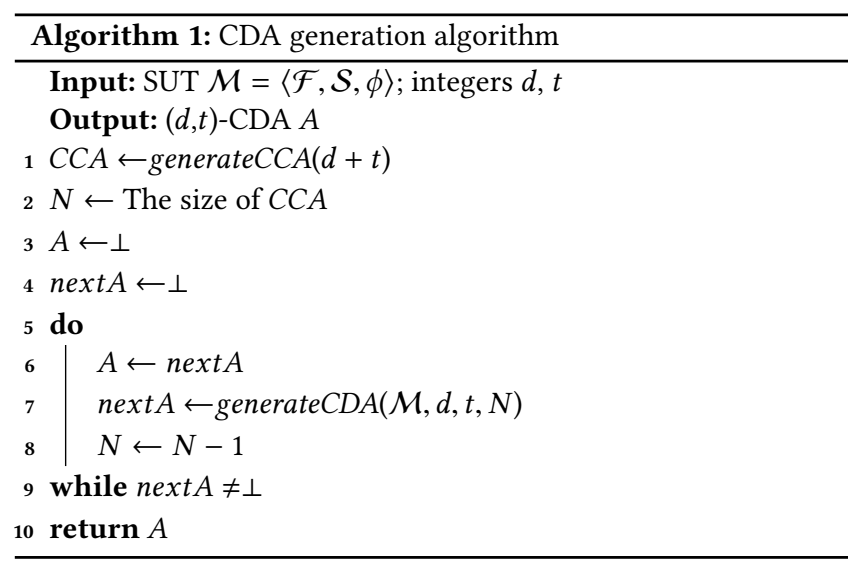

The domain of $p_{i}$ is $\left\{0,1, \ldots,\left|S_{i}\right|-1\right\}$. Note that $S_{i}$ is the domain of parameter $F_{i}$.

Enumerating Valid Interactions Obtaining $\mathcal{V} \mathcal{I}_{t}$ means enumerating all valid interactions of strength $t$. This can be performed by checking every $t$-way interaction against whether it is valid or not. Recall that an interaction is valid if and only if it can be covered by a valid test case; that is:

$$
\exists \sigma \in \mathcal{R}: T \subseteq \sigma .
$$

Similar to ValidTest( $n$ ), the following expression can be used to check the validity of a given interaction $T=\left\{\left(F_{i_{1}}, v_{i_{1}}\right), \ldots,\left(F_{i_{t}}, v_{i_{t}}\right)\right\}$ $\left(1 \leq i_{1} \leq i_{t} \leq k\right)$.

$$
\operatorname{checkValidity}(T):=\left.\bigwedge_{j=1}^{t}\left(p_{i_{j}}=v_{i_{j}}\right) \wedge \bigwedge_{m=1}^{|\phi|} \phi_{m}\right|_{p_{1}, p_{2}, \ldots, p_{k}}
$$

If this expression is satisfiable, then the interaction $T$ is valid; otherwise, it is invalid. The set $\mathcal{V} \mathcal{I}_{t}$ can be obtained by checking the satisfiability of the above formula for all $t$-way interactions.

Enumerating $\mathcal{T}-T$ Pairs Now consider enumerating all $\mathcal{T}-T$ pairs such that $T \in \mathcal{V} \mathcal{I}_{t}, \mathcal{T} \subseteq \mathcal{V} \mathcal{I}_{t},|\mathcal{T}|=d, T \notin \mathcal{T}$, and $\mathcal{T} \ngtr T$. Since we can assume that $\mathcal{V} I_{t}$ has been obtained by the process described above, the problem that matters is how to decide whether or not $\mathcal{T}\left(\subseteq \mathcal{V} \mathcal{I}_{t}\right)$ masks $T\left(\in \mathcal{V} \mathcal{I}_{t}\right)$ when $\mathcal{T}$ and $T \notin \mathcal{T}$ are given

By the definition of masking, given such a $\mathcal{T}-T$ pair, $\mathcal{T}$ does not mask $T$ if and only if the following condition holds:

$$
\exists \boldsymbol{\sigma} \in \mathcal{R}: T \subseteq \sigma \wedge \neg\left(\exists T^{\prime} \in \mathcal{T}: T^{\prime} \subseteq \sigma\right)
$$

In words, the condition holds if there is a valid test case that covers the interaction $T$ but does not cover any interactions in the interaction set $\mathcal{T}$. Hence, given $\mathcal{T}$ and $T, \mathcal{T} \nsucc T$ holds if and only if the following expression evaluates to true.

$$
\begin{aligned}
& \operatorname{checkUnMasking}(\mathcal{T}, T):= \\
& \left.\bigwedge_{j=1}^{t}\left(p_{x_{j}}=v_{x_{j}}\right) \wedge \neg\left(\bigvee_{L=1}^{d} \bigwedge_{l=1}^{t}\left(p_{y_{L_{l}}}=v_{y_{L_{l}}}\right)\right) \wedge \bigwedge_{m=1}^{|\phi|} \phi_{m}\right|_{p_{1}, p_{2}, \ldots, p_{k}}
\end{aligned}
$$

where $\mathcal{T}=\left\{\left\{\left(F_{y_{1_{1}}}, v_{y_{1_{1}}}\right), \ldots,\left(F_{y_{1 t}}, v_{y_{1_{t}}}\right)\right\}, \ldots,\left\{\left(F_{y_{d_{1}}}, v_{y_{d_{1}}}\right), \ldots\right.\right.$, $\left.\left.\left(F_{y_{d_{t}}}, v_{y_{d_{t}}}\right)\right\}\right\}$ and $T=\left\{\left(F_{x_{1}}, v_{x_{1}}\right), \ldots,\left(F_{x_{t}}, v_{x_{t}}\right)\right\}$.

\subsection{Algorithm for Generating $(d, t)$-CDAs}

Algorithm 1 repeatedly solves the problem of finding a $(d, t)$-CDA while varying the array size $N$. The array size $N$ starts with a value large enough to ensure the existence of a CDA and is gradually decreased until no existence of a CDA of size $N$ is proved. To obtain the initial value of $N$, the algorithm creates a $(d+t)$-CCA using an off-the-shelf algorithm (line 1), where the CCA generation algorithm is represented as function generateCCA $(x)$ which returns an $x$-CCA. Our algorithm uses the size of the CCA as the initial $N$. This idea comes from our conjecture that a $(d+t)$-CCA is already a $(d, t)$-CDA. (We leave the proof for future work.)

In the algorithm, generate $C D A(\mathcal{M}, d, t, N)$ in line 7 represents a function that produces a $(d, t)$-CDA of size $N$ by checking the satisfiability of expression (12), as described in Section 4.1. If the expression is satisfiable, then the SMT solver returns SAT as well as the satisfying assignment of values to variables. In this case, a $(d, t)$-CDA of size $N$ is obtained, since the satisfying assignment represents the $(d, t)$-CDA. The size $N$ is decreased by one and the same process is repeated. If the result of satisfiability check is UNSAT (unsatisfiable), it is proved that no CDA of size $N$ exists (denoted as $\perp$ in the algorithm). Then the algorithm returns the CDA of size $N+1$ and stops its execution.

One might think that binary search could work better to vary $N$ than the linear search adopted by the algorithm. In fact, this is not the case because showing unsatisfiability, that is, the nonexistence of a CDA, usually takes much longer time than showing satisfiability, that is, the existence of a CDA. The linear search delays solving an unsatisfiable expression until all possible sizes are checked, avoiding getting trapped in a long computation required for the unsatisfiable problem instance.

\section{EXPERIMENTS}

We wrote a program in the $\mathrm{C}++$ language that implemented the proposed algorithm and conducted some experiments by applying the program to well-known benchmark problems. ${ }^{1}$ There are many benchmark models in the CTWedge repository associated with [7]. We applied our method to small-scale benchmarks, mainly from [21] and [13], in the repository. Since testing of two-way interactions is the most common form of Combinatorial Interaction Testing (CIT), we consider the case $t=2$. Also, we assume that the number of faulty interactions is at most one, that is, $d=1$.

All the experiments were conducted on a machine with Intel Core i7-8700 CPU, 64GB memory and Ubuntu 18.04LTS OS. In order to obtain the initial array size, the algorithm first computes a $(d+t)$-CCA. For this purpose, we use the CIT-BACH tool (version 1.10) [26]. This tool implements a heuristic algorithm that uses random numbers, and thus the output CCA can be different for different runs. Hence, in order to obtain a single CCA, we ran it five times and chose the smallest one of the five CCAs obtained. Our program uses the Z3 SMT solver library for satisfiability checking [5]. For each run of the program, we set the timeout period to 30 minutes.

Experimental results are shown in Table 6. We only list the results for the instances for which the proposed method succeeded in generating CDAs. The two leftmost columns show the IDs and

\footnotetext{
${ }^{1}$ The source code is available at https://github.com/k-kou/CDAGenbySMT
} 
Table 6: Experimental Results

\begin{tabular}{c|c|c|c|c|c|cc|cc}
\hline \multirow{2}{*}{ No. } & \multirow{2}{*}{ SUT } & $\mathcal{F}$ & $|\phi|$ & $\left|\mathcal{V} \mathcal{I}_{2}\right|$ & $|\{\mathcal{T}>T\}|$ & \multicolumn{2}{|c|}{ Size } & \multicolumn{2}{|c}{ Time (s) } \\
\cline { 5 - 8 } & & & & & & & \\
& & & & & & \\
& Concurrency & $2^{5}$ & 7 & 36 & 130 & 8 & 8 & 0.21 & 0.23 \\
2 & Storage1 & $2^{1} 3^{1} 4^{1} 5^{1}$ & 95 & 53 & 112 & 25 & 25 & 1.05 & T.O. \\
3 & Banking1 & $3^{4} 4^{1}$ & 111 & 102 & 0 & 60 & 24 & $1,324.29$ & T.O. \\
4 & Car & $2^{9}$ & 15 & 102 & 1,487 & 13 & 12 & 4.94 & 169.05 \\
5 & Storage2 & $3^{4} 6^{1}$ & 0 & 126 & 0 & 77 & 53 & $1,471.80$ & T.O. \\
6 & Movies_App & $2^{13}$ & 23 & 211 & 4,968 & 11 & 10 & 13.86 & 49.20 \\
7 & Aircraft & $2^{13}$ & 19 & 239 & 2,647 & 24 & 14 & 395.55 & T.O. \\
8 & REAL_12 & $2^{14}$ & 23 & 275 & 5,368 & 33 & 28 & 828.68 & T.O. \\
9 & Comm_Protocol & $2^{10} 7^{1}$ & 127 & 285 & 2,177 & 54 & 54 & 1137.43 & T.O. \\
10 & Stack & $2^{17}$ & 28 & 465 & 6,399 & 45 & 43 & 1457.51 & T.O. \\
11 & Graph_Product_Line & $2^{20}$ & 45 & 499 & 37,212 & 27 & 26 & 1353.48 & T.O. \\
12 & Connector & $2^{20}$ & 37 & 537 & 49,038 & 18 & 18 & 517.14 & T.O. \\
\hline
\end{tabular}

For the problems in bold font (eg. Car), the proposed algorithm was able to generate optimal CDAs within the 30 minutes time limit.

names of the benchmark problems. The column labeled $\mathcal{F}$ shows the domain sizes of all parameters in the exponential format. For example, $3^{4} 4^{1}$ for Banking 1 means that there is a total of five parameters and four of them have a domain of size 3 and one has a domain of size 4 . The $|\phi|$ column shows the number of constraints. The column labeled $\left|\mathcal{V} \mathcal{I}_{2}\right|$ shows the total number of valid two-way interactions, while the column labeled $|\{\mathcal{T}>T\}|$ represents the total number of $\mathcal{T}-T$ pairs such that $\mathcal{T}>T$.

The two columns labeled Size show the size of the CCA, i.e., the initial array size, and the size of the smallest CDA that was generated in the course of the program execution. It should be noted that the algorithm repeatedly constructs CDAs of different sizes in a single run, and thus some CDA was obtained even when the program timed out.

The SAT column shows the time to receive the last SAT outcome from the SMT solver, that is, the running time required to obtain the smallest CDA before completion or timeout. The UNSAT column shows the time to receive the UNSAT outcome, that is the whole running time of the program. T.O. means that the program reached the 30 minute timeout limit before completion.

For Concurrency, Car and Movies_App, the program was successful in completing its execution within the timeout limit. Therefore, the CDAs obtained for these problems are optimal in size. The program timed out for the other problems; but it was able to generate some CDAs for all the problems.

Basically, the satisfiability checking becomes increasingly difficult as the array size $N$ reaches the minimum (optimal). All the three optimal CDAs obtained consist of a very small number of rows (test cases), and thus the satisfiability problem was relatively easy to solve even when the array size $N$ was optimal. For the same reason, even when the number of rows, that is, the array size is not small, finding a CDA of that size can be easy if the size is fairly larger than the minimum one. This explains the results where even when the algorithm execution was not completed, at least some $(1,2)$-CDA was always obtained during the execution.

\section{RELATED WORKS}

\subsection{Locating Faulty Interactions}

In addition of DAs, there also exist a few means for fault localization in Combinatorial Interaction Testing (CIT). Examples include Minimal Failure-Causing Schemas (MFSs) [19], Locating Arrays (LAs) [3], Constrained Locating Arrays (CLAs) [12].

The MFS-based testing strategy, proposed by Nie et al., does not require a prior knowledge or assumption about the number $d$ of faulty interactions and the strength $t$ of faulty interactions. Unlike the DA or CDA-based strategies, the MFS-based strategy uses follow-up tests to locate faulty interactions. In other words, based on the obtained test outcome, a set of new test cases are designed and executed in order to gain further information on faulty interactions.

The DA or CDA-based strategies do not need to execute additional test cases. Locating Arrays (LAs) are mathematical objects similar to DAs, and CLAs are an extension of LAs that incorporates constraints. As the DA-based strategies, no additional test cases are required when using LAs or CLAs to identify faulty interactions. When compared to DAs and CDAs, LAs and CLAs can be constructed using fewer test cases, thus resulting in less testing cost. However, LAs and CLAs may lead to incorrect fault localization if there are more than $d$ faulty interactions. In contrast, as mentioned in the previous sections, when there are more than $d$ faulty interactions, DAs and CDAs are able to detect this situation, thus avoiding incorrect fault localization.

\subsection{Research on Detecting Arrays}

In this paper, we proposed CDAs to extend DAs to incorporate constraints. Another interesting extension was done by Colbourn et al. [4, 22] aiming to deal with unreliable test outcomes. They introduced a new parameter $\delta$ to quantify the tolerance to unreliable test outcomes. Specifically, the value of $\delta$ represents the maximum number of unreliable test cases that a DA can tolerate without causing incorrect fault localization. Other studies on DAs investigated mathematical properties of DAs, such as the bounds on array size or classes of optimal DAs and the conditions required for their existence [23-25]. 


\subsection{Methods for Test Suite Generation}

In the field of CIT, many approaches have been proposed to generate test suites. Most of them are aimed to generate Covering Arrays (CAs) or Constrained Covering Arrays (CCAs) and many of them use some heuristic techniques. AETG [1] is one of the best known systems for test suite generation for CIT. This system adopts the one-test-at-a-time greedy strategy for generating CCAs. The one-test-at-a-time greedy strategy builds a whole test suite by repeatedly adding a test case that covers most interactions that are yet to be covered. Another type of a greedy strategy is the one-parameter-at-a-time greedy strategy. In this strategy, a CCA for the first $t$ parameters is constructed first. Then, the CCA is repeatedly extended to include one of the remaining parameters until all parameters are incorporated. For example, an algorithm named IPOG [17] adopts this strategy. Metaheuristic algorithms, such as simulated annealing (e.g. [8, 9]) and tabu search (e.g., [6]) have also been investigated for the purpose of constructing small CCAs.

Satisfiability-based constructions of CAs, CCAs, LAs, and CLAs can be found in $[10,11,14,18]$.

\section{CONCLUSIONS}

When constraints exist among test parameters, Detecting Arrays (DAs) cannot be directly used as test suites, because it may be impossible to form DAs with constraint-satisfying test cases. This paper proposed Constrained Detecting Arrays (CDAs), a new type of DAs which can be applied to SUTs with constraints. To this end, the notion of masking was introduced to formally capture how constraints restrict satisfying the requirement of DAs. This notion was used to relax the fault localizing requirement of DAs, thus leading to the definition of CDAs. Also a computational method was proposed to generate CDAs. The proposed method reduces the problem of generating a CDA to the satisfiability problem and then solves the problem using an SMT solver. Experimental results showed that the method is able to generate CDAs for practical problem instances within reasonable time.

In future work, we plan to develop different methods of constructing CDAs. We are particularly interested in heuristic approaches because sub-optimal (i.e., sub-minimum) CDAs, instead of optimal ones, are usually sufficient for practical usages. In fact, many heuristic approaches have been developed for construction of CCAs. We plan to adapt some of these approaches to the generation of CDAs.

\section{REFERENCES}

[1] David M. Cohen, Siddhartha R. Dalal, Michael L. Fredman, and Gardner C. Patton 1997. The AETG System: An Approach to Testing Based on Combinatiorial Design. IEEE Trans. Software Eng. 23, 7 (1997), 437-444.

[2] M. B. Cohen, M. B. Dwyer, and J. Shi. 2008. Constructing Interaction Test Suites for Highly-Configurable Systems in the Presence of Constraints: A Greedy Approach. IEEE Trans. Software Eng. 34, 5 (Sept 2008), 633-650.

[3] Charles J. Colbourn and Daniel W. McClary. 2008. Locating and detecting arrays for interaction faults. Fournal of combinatorial optimization 15, 1 (2008), 17-48.

[4] Charles J. Colbourn and Violet R. Syrotiuk. 2019. Detecting Arrays for Main Effects. In Algebraic Informatics - 8th International Conference, CAI 2019, Niš, Serbia, fune 30 - July 4, 2019, Proceedings. 112-123.

[5] Leonardo De Moura and Nikolaj Bjørner. 2008. Z3: An Efficient SMT Solver. In Proceedings of the Theory and Practice of Software, 14th International Conference on Tools and Algorithms for the Construction and Analysis of Systems (TACAS'08/ETAPS'08). Springer-Verlag, Berlin, Heidelberg, 337-340.

[6] Philippe Galinier, Segla Kpodjedo, and Giulio Antoniol. 2017. A Penalty-based Tabu Search for Constrained Covering Arrays. In Proceedings of the Genetic and
Evolutionary Computation Conference (GECCO '17). ACM, New York, NY, USA, 1288-1294. https://doi.org/10.1145/3071178.3071324

[7] A. Gargantini and M. Radavelli. 2018. Migrating Combinatorial Interaction Test Modeling and Generation to the Web. In 2018 IEEE International Conference on Software Testing, Verification and Validation Workshops (ICSTW). 308-317. https://doi.org/10.1109/ICSTW.2018.00066

[8] B. J. Garvin, M. B. Cohen, and M. B. Dwyer. 2009. An Improved Meta-heuristic Search for Constrained Interaction Testing. In 2009 1st International Symposium on Search Based Software Engineering. 13-22. https://doi.org/10.1109/SSBSE.2009.25

[9] Brady J. Garvin, Myra B. Cohen, and Matthew B. Dwyer. 2011. Evaluating improvements to a meta-heuristic search for constrained interaction testing. Empirical Software Engineering 16, 1 (2011), 61-102.

[10] Brahim Hnich, Steven Prestwich, and Evgeny Selensky. 2005. Constraint-Based Approaches to the Covering Test Problem. In Recent Advances in Constraints, Boi V. Faltings, Adrian Petcu, François Fages, and Francesca Rossi (Eds.). Springer Berlin Heidelberg, Berlin, Heidelberg, 172-186.

[11] H. Jin, T. Kitamura, E. Choi, and T. Tsuchiya. 2018. A Satisfiability-Based Approach to Generation of Constrained Locating Arrays. In 2018 IEEE International Conference on Software Testing, Verification and Validation Workshops (ICSTW). 285-294. https://doi.org/10.1109/ICSTW.2018.00062

[12] Hao Jin and Tatsuhiro Tsuchiya. 2018. Constrained locating arrays for combinatorial interaction testing. CoRR abs/1801.06041 (2018), 1-12. arXiv:1801.06041 http://arxiv.org/abs/1801.06041

[13] Martin Fagereng Johansen, Øystein Haugen, Franck Fleurey, Anne Grete Eldegard, and Torbjørn Syversen. 2012. Generating Better Partial Covering Arrays by Modeling Weights on Sub-product Lines. In Model Driven Engineering Languages and Systems, Robert B. France, Jürgen Kazmeier, Ruth Breu, and Colin Atkinson (Eds.). Springer Berlin Heidelberg, Berlin, Heidelberg, 269-284.

[14] T. Konishi, H. Kojima, H. Nakagawa, and T. Tsuchiya. 2017. Finding Minimum Locating Arrays Using a SAT Solver. In 2017 IEEE International Conference on Software Testing, Verification and Validation Workshops. 276-277. https://doi.org/ 10.1109/ICSTW.2017.49

[15] D. Richard Kuhn, Raghu N. Kacker, and Yu Lei. 2013. Introduction to combinatorial testing. CRC Press.

[16] D. Richard Kuhn and Daniel R. Wallace. 2004. Software fault interactions and implications for software testing. IEEE Transactions on Software Engineering 30, 6 (June 2004), 418-421.

[17] Yu Lei, Raghu Kacker, D. Richard Kuhn, Vadim Okun, and James Lawrence. 2008. IPOG/IPOG-D: efficient test generation for multi-way combinatorial testing. Software Testing, Verification and Reliability 18, 3 (2008), 125-148. https://doi.org/ 10.1002/stvr.381 arXiv:https://onlinelibrary.wiley.com/doi/pdf/10.1002/stvr.381

[18] Toru Nanba, Tatsuhiro Tsuchiya, and Tohru Kikuno. 2012. Using Satisfiability Solving for Pairwise Testing in the Presence of Constraints. IEICE Transactions on Fundamentals of Electronics, Communications and Computer Sciences E95.A, 9 (2012), 1501-1505. https://doi.org/10.1587/transfun.E95.A.1501

[19] Changhai Nie and Hareton Leung. 2011. The Minimal Failure-Causing Schema of Combinatorial Testing. ACM Trans. Softw. Eng. Methodol. 20, 4, Article 15 (Sept. 2011), 38 pages. https://doi.org/10.1145/2000799.2000801

[20] Changhai Nie and Hareton Leung. 2011. A Survey of Combinatorial Testing. ACM Comput. Surv. 43, 2, Article 11 (Feb. 2011), 29 pages. https://doi.org/10. $1145 / 1883612.1883618$

[21] Itai Segall, Rachel Tzoref-Brill, and Eitan Farchi. 2011. Using binary decision diagrams for combinatorial test design. In Proc. of the 2011 International Symposium on Software Testing and Analysis (ISSTA). ACM, 254-264.

[22] Stephen A. Seidel, Kaushik Sarkar, Charles J. Colbourn, and Violet R. Syrotiuk. 2018. Separating Interaction Effects Using Locating and Detecting Arrays. In Combinatorial Algorithms - 29th International Workshop, IWOCA 2018, Singapore, July 16-19, 2018, Proceedings. 349-360. https://doi.org/10.1007/978-3-319-94667229

[23] Ce Shi, Yu Tang, and Jianxing Yin. 2014. Optimum mixed level detecting arrays. The Annals of Statistics 42 (08 2014). https://doi.org/10.1214/14-AOS1228

[24] Ce Shi and Cheng Min Wang. 2016. Optimum detecting arrays for independent interaction faults. Acta Mathematica Sinica, English Series 32, 2 (01 Feb 2016), 199-212. https://doi.org/10.1007/s10114-016-5049-7

[25] Yu Tang and Jian Xing Yin. 2011. Detecting arrays and their optimality. Acta Mathematica Sinica, English Series 27, 12 (01 Dec 2011), 2309-2318. https://doi. org/10.1007/s10114-011-0184-7

[26] Tatsuhiro Tsuchiya. 2019. Using binary decision diagrams for constraint handling in combinatorial interaction testing. CoRR abs/1907.01779 (2019). arXiv:1907.01779 http://arxiv.org/abs/1907.01779

[27] Huayao Wu, Nie Changhai, Justyna Petke, Yue Jia, and Mark Harman. 2019. Comparative Analysis of Constraint Handling Techniques for Constrained Combinatorial Testing. IEEE Transactions on Software Engineering PP (11 2019), 1-1.

[28] Huayao Wu, Changhai Nie, Justyna Petke, Yue Jia, and Mark Harman. 2019. A Survey of Constrained Combinatorial Testing. CoRR abs/1908.02480 (2019). arXiv:1908.02480 http://arxiv.org/abs/1908.02480 